\title{
NOTA
}

\section{ALTERAÇÕES NA FERTILIDADE DO SOLO EM SISTEMAS DE ROTAÇÃO DE CULTURAS EM SEMEADURA DIRETA ${ }^{(1)}$}

\author{
J . C. FRANCHINI (2), C. M. BORKERT (3), \\ M. M. FERREIRA(4) \& C. A. GAUDÊ NCIO(5)
}

\begin{abstract}
RESUMO
O sistema de semeadura direta com culturas de grãos ocupa atualmente 2,5 milhões de hectares no estado do Paraná. Além do sistema tradicional de sucessão trigo-soja, em grande parte desta área são semeadas plantas de cobertura-adubação verde no inverno e milho no verão. Neste estudo, foram avaliadas as alterações em algumas características químicas do solo, após sete anos, sob os sistemas de rotação de culturas trigo-soja-aveia-soja (TSA), trigosoja-tremoço-milho-aveia-soja (TMA), tremoço-milho-trigo-soja (TM) e o sistema tradicional de sucessão trigo-soja (TS) em unidades de Latossolo Roxo distrófico, em Londrina e Campo Mourão. O sistema TM diminuiu o pH do solo e os teores de cálcio $\left(\mathrm{Ca}^{2+}\right)$ e magnésio $\left(\mathrm{Mg}^{2+}\right)$ trocáveis e aumentou o alumínio trocável $\left(\mathrm{Al}^{3+}\right)$, a acidez potencial $(\mathrm{H}+\mathrm{Al})$ e o $\mathrm{N}$-total do solo em relação ao sistema TS. E ssas caracaterísticas apresentaram valores intermediários nos demais sistemas. Não foram observadas diferenças entre os sistemas quanto aos teores de carbono orgânico e potássio trocável $\left(\mathrm{K}^{+}\right)$no solo. A di minuição do teor de $\mathrm{Ca}^{2+}$ no sistema TM foi maior do que a quantidade de Ca aplicado na forma de calcário durante o experimento. A adubação nitrogenada no trigo e no milho foi relacionada com a acidificação observada no solo. A manutenção dos níveis de $\mathrm{K}^{+}$e di minuição dos níveis de $\mathrm{Ca}^{2+}$ e $\mathrm{Mg}^{2+}$ resultaram provavelmente de uma alteração na preferência de lixiviação de cátions no sistema TM. A formação de complexos orgânicos com cátions divalentes foi sugerida como provável mecanismo responsável por estas alterações químicas no solo.
\end{abstract}

Termos de indexação: acidificação do solo, mobilidade de cátions, plantas de cobertura, adubação verde, cálcio.

(1) Trabalho enviado para publicação em setembro de 1998 e aceito para publicação em fevereiro de 2000.

(2) Engenheiro-Agrônomo, Embrapa Soja. Caixa Postal 231, CEP 86001-970 Londrina (PR). Bolsista do CNPq.

(3) Engenheiro-Agrônomo, Embrapa Soja. Bolsista do CNPq.

(4) Professor Adjunto do Departamento de Solos e Nutrição de Plantas, Universidade Federal de Lavras - UFLA. Caixa Postal 37, CEP 37200-000 Lavras (MG).

(5) Engenheiro-Agrônomo, MSc. Embrapa Soja. 


\title{
SUMMARY: SOIL FERTILITY CHANGES ON NO-TILLAGE CROP ROTATION SYSTEMS
}

\begin{abstract}
Theno-tillage system with grain crops occupies today 2.5 millions hectares in thestate of Paraná. Besides the traditional rotation system with wheat and soybean, in many areas green manurecover crops arealso sown in theWinter and corn in theSummer. Theobjective of this study was to eval uate changes in someof thesoil chemical properties after eight years under the crop rotation systems: wheat-soybean-black oat-soybean (WOS), wheat-soybeanI upine-corn-black oat-soybean ( LCO), I upinecorn-wheat-soybean (LC) and compare them with the traditional system wheat-soybean (WS) grown in two Oxisols at Londrina and Campo Mourão, State of Paraná, Brazil. The LC crop rotation system reduced the soil pH and the level of exchangeable calcium $\left(\mathrm{Ca}^{2+}\right)$ and magnesium $\left(\mathrm{Mg}^{2+}\right)$ and increased the excangeable aluminum $\left(\mathrm{Al}^{3+}\right)$, the potential acidity $(\mathrm{H}+\mathrm{Al})$, and the total- $\mathrm{N}$ in the soil as compared to theWS system. These characteristics showed intermediate values in the other systems. There were no significant differences on soil levels of organic carbon and exchangeable $\mathrm{K}\left(\mathrm{K}^{+}\right)$among the crop rotation systems. The decrease on the level of $\mathrm{Ca}^{2+}$ in the LC system was larger than the amount of $\mathrm{Ca}$ applied as lime to the soil during the experiment. Soil acidification was related to the nitrogen fertilization of wheat and corn. Themaintenance of level s of $\mathrm{K}^{+}$and the decreaseon thelevel s of $\mathrm{Ca}^{2+}$ and $\mathrm{Mg}^{2+}$ demonstrate that therewas a changein the preference of leaching cations in the LC crop rotation system. The formation of metal-organic complexes with divalent cations was assumed to be the possible mechanism responsible for the chemical alterations in the soil.
\end{abstract}

Index terms: soil acidification, cation mobility, cover crops, green manure, calcium, crop rotation.

\section{NTRODUÇÃO}

O sistema de semeadura direta (SD) tem sido cada vez mais explorado em todo o Brasil. Particularmente no Paraná, onde o sistema foi instalado na década de 70, mais de 2,5 milhões de hectares são cultivados atual mente sob este sistema. O sucesso da SD como sistema de produção agrícola está diretamente relacionado com as alterações observadas na dinâmica de decomposição dos resíduos vegetais, considerando o revolvimento do solo.

Os resíduos vegetais mantidos na superfície do sol o funcionam como um reservatório de nutrientes que são liberados lentamente pela ação de microrganismos, aumentam a estabilidade estrutural e protegem contra a erosão hídrica (Kemper \& Derpsch, 1981; Sidiras et al., 1982). Com o passar do tempo, ocorre um aumento no teor de matéria orgânica do solo (MOS) devidoà menor taxa de decomposição dos resíduos vegetais, o que aumenta a fertilidade de solos ácidos com cargas dependentes de $\mathrm{pH}$ associadas à matéria orgânica, predominantes no território brasileiro (Pavan et al., 1985; Sidiras \& Pavan, 1985; Rheinheimer et al., 1998).

A menor taxa de decomposição da MOS pode acarretar, nos primeiros anos de cultivo sob SD, deficiência de $\mathrm{N}$ em culturas exigentes como o milho.
A longo prazo, o aumento do teor de MOS conduz ao aumento do reservatório de $\mathrm{N}$ potencialmente mineralizável, compensando a menor taxa de decomposição. Este processo pode ser acelerado em sistemas de rotação de culturas envolvendo leguminosas (Muzilli et al., 1983; Heinzmann, 1985; Derpsch et al., 1985; Bayer \& Mielniczuk, 1997; Rheinheimer et al., 1998).

A ausência de revolvimento do solo na SD também implica a não-incorporação do calcário aplicado na superfície do solo. Neste caso, o pH e os teores de $\mathrm{Ca}$ e $\mathrm{Mg}$ somente seriam aumentados nas camadas superficiais do sol o. Esta restrição do efeito al calino do cal cário ao local de aplicação é atribuída à sua baixa solubilidade, à ausência de um ânion estável em sua reação no sol o e à geração de cargas dependentes de pH (Gonzalez-Erico et al., 1979; Ritchey et al., 1982; Pavan et al., 1984). Entretanto, no sistema de SD, a presença de resíduos vegetais na superfície proporciona um aumento dos níveis de $\mathrm{pH}$ e dos teores de Ca e Mg trocáveis até camadas mais profundas do solo em detrimento do teor de Al trocável (Sidiras \& Pavan, 1985, Oliveira \& Pavan, 1996; Pöttker \& Ben, 1998; Caires et al., 1998; 1999).

Em sistemas de rotação de culturas intensivos, utilizando plantas de cobertura-adubação verde no inverno, existem poucas informações sobre seus efeitos no teor de $\mathrm{N}$ total do sol o e no comportamento do calcário aplicado na superfície do solo em 
experimentos de longa duração. Deste modo, o objetivo do presente estudo foi avaliar, após seteanos em sistema de SD, as alterações químicas de Latossolos Roxos da região norte do Paraná corrigidos com cal cário e submetidos a sistemas de rotação de culturas com plantas de coberturaadubação verde.

\section{MATE RIAL E MÉTODOS}

Os experimentos avaliados neste estudo foram instalados, em 1985, no campo experimental da Embrapa - Centro Nacional de Pesquisa deSoja, em Londrina, ena fazenda experimental da Cooperativa Agropecuária Mourãoense, em Campo Mourão. Em ambos os locais, o solo é classificado como L atossolo Roxo distrófico (LRd) muitoargil oso. Os teores ( $\mathrm{g} \mathrm{kg}^{-1}$ ) deargila, silte eareia, na camada de $0-20 \mathrm{~cm}$, foram, respectivamente, de $710,170,120$, em L ondrina, e de 710, 220 e 70, em Campo Mourão. Ambas as áreas, até o verão de 1985, vinham sendo cultivadas com soja no verão e trigo no inverno em sistema de preparo convencional, isto é, revolvimento do solo na camada de $0-20 \mathrm{~cm}$ com arado de discos, desde 1970 (em Campo M ourão) e 1973 (em Londrina). As dimensões das parcelas eram de $30 \times 6 \mathrm{~m}$, em Campo Mourão, ede 14 × 4 m, em Londrina. O delineamento experimental utilizado foi o de blocos casualizados, com quatro repetições. Os tratamentos são apresentados no quadro 1.
Em março de 1985, para instalação dos experimentos, as áreas foram corrigi das com cal cário dol omítico numa dose equivalenteà necessária para atingir $60 \%$ da saturação por bases, o que correspondeu a $2.500 \mathrm{~kg} \mathrm{ha}^{-1}$ (Campo Mourão) e $2.000 \mathrm{~kg} \mathrm{ha}^{-1}$ (Londrina). O cal cário foi incorporado com arado de discos até à profundidade de $20 \mathrm{~cm}$. No início do sexto ano do experimento, em 1991, antes da implantação da cultura de verão, no caso a soja, todos os tratamentos receberam nova dose de cal cário, tendo como objetivo a mesma saturação por bases a ser atingida (60\%), o que correspondeu a $1.700 \mathrm{~kg} \mathrm{ha}^{-1}$, em Campo Mourão, e $2.000 \mathrm{~kg} \mathrm{ha}^{-1} \mathrm{em}$ L ondrina. N esta oportunidade, o cal cário foi aplicado na superfície do solo, sem ser incorporado. As análises químicas ( $\mathrm{pH}, \mathrm{Al}, \mathrm{K}, \mathrm{Ca}, \mathrm{Mg}, \mathrm{H}+\mathrm{Al}$ e C orgânico) da camada de 0-20 cm dos solos, após a calagem realizada na instalação dos experimentos, foram tomadas como referência para avaliar as alterações químicas após sete anos de condução do experimento em rotação de culturas (Figuras 1 e 2 , linha tracejada).

Em ambos os experimentos, as culturas receberam as seguintes adubações (em kg ha-1): (a) trigo (Triticum aestivum): 20 de N, 60 de $\mathrm{P}_{2} \mathrm{O}_{5}$ e 20 de $\mathrm{K}_{2} \mathrm{O}$, na semeadura, mais 20 de $\mathrm{N}$, em cobertura; (b) milho (Zea mays): 30 de N, 50 de $\mathrm{P}_{2} \mathrm{O}_{5}$ e 50 de $\mathrm{K}_{2} \mathrm{O}$, na semeadura, mais 40 de $\mathrm{N}$, em cobertura; (c) soja (Glicinemax): 40 de $_{2} \mathrm{O}_{5}$ e 40 de $\mathrm{K}_{2} \mathrm{O}$, na semeadura. $\mathrm{O}$ nitrogênio na semeadura e em cobertura foi aplicado na forma de sulfato de amônio. As plantas de cobertura-adubação verde,

Quadro 1. Seqüência de culturas e método de semeadura nos diferentes sistemas de rotação de culturas avaliados em Londrina e Campo Mourão

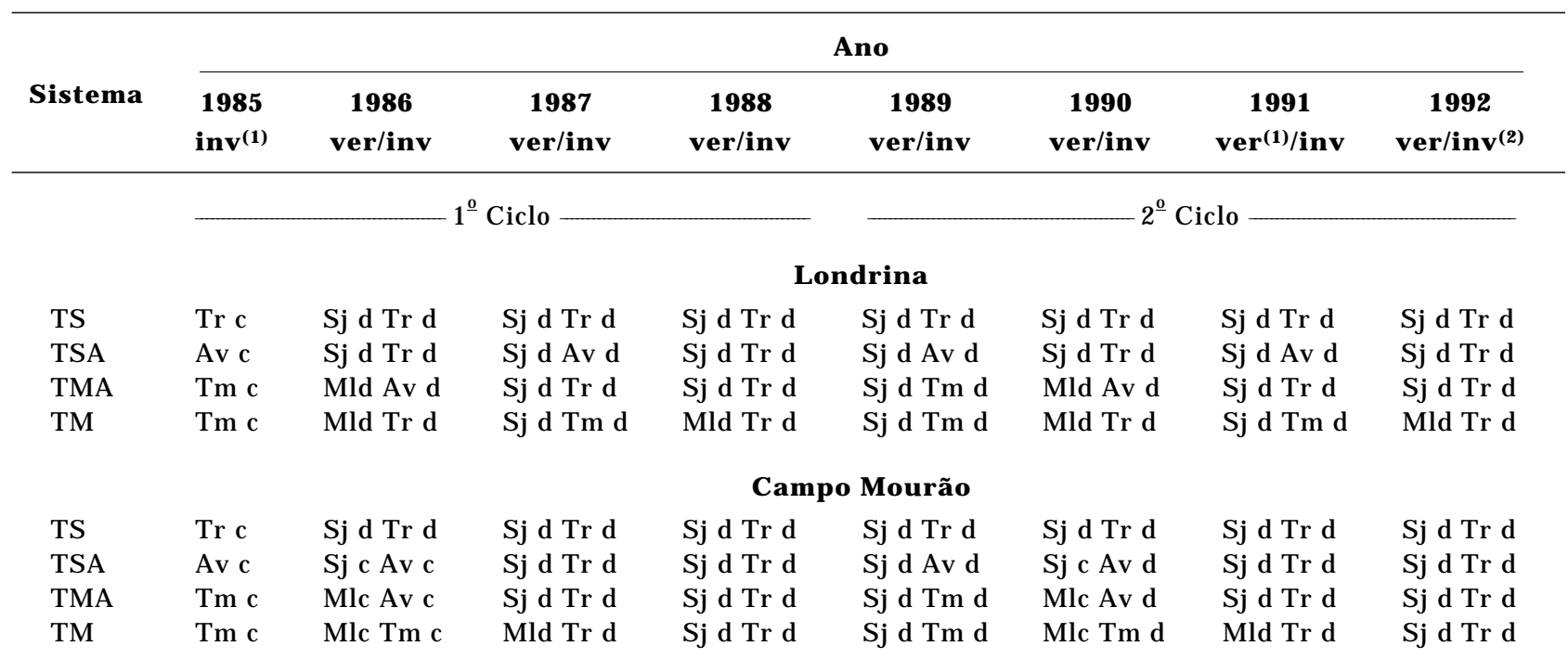

Tm: tremoço, Sj: soja, Tr: trigo, Av: aveia, Ml: milho; c: plantio convencional; d: semeadura direta; inv: inverno; ver: verão. ${ }^{(1)}$ aplicação de calcário. (2)amostragem de solo após o trigo. 
tremoço azul (Lupinus angustifolius) e aveia preta (Avena strigosa) não receberam adubação. Para controlar as plantas invasoras, foram utilizados Sethoxidin e Fomezafen para gramíneas e folhas largas, respectivamente. A soja foi semeada no espaçamento de $45 \mathrm{~cm}$ entrel inhas e população final de 400.000 plantas ha-1. Para o trigo, milho, aveia e tremoço, esses valores foram de $18 \mathrm{~cm}$ e 4.500.000 plantas ha-1; 90 cm e 50.000 plantas ha-1; $18 \mathrm{~cm}$ e 3.000 .000 plantas ha-1; $45 \mathrm{~cm}$ e 300.000 plantas ha-1, respectivamente. As plantas de cobertura-adubação verde (tremoço e aveia), quando atingiram o estádio de grão leitoso, foram cortadas pela passagem de rolo faca. A semeadura direta foi o sistema predominante em ambos os experimentos, embora, em Campo Mourão, o plantio convencional tenha sido utilizado em algumas oportunidades (Quadro 1).

A amostragem de sol o foi realizada em 1992, após sete anos de cultivo sob os diferentes sistemas de rotação de culturas iniciados em 1985 (Quadro 1), sendo na última semana de setembro em Campo Mourão e na primeira semana de novembro em Londrina. Todas as parcelas sob cultivo foram amostradas após a col heita do trigo (Quadro 1). No centro de cada parcela, foi aberta uma trincheira, perpendicularmente ao sentido das linhas de semeadura, com $0,5 \mathrm{~m}$ de profundidade, 1,0 de largura e 1,0 de comprimento, na qual foram tomadas amostras nas seguintes camadas: 0-10, 1020 e $20-40 \mathrm{~cm}$. O material orgânico presente na superfície foi retirado manualmente e o solo compreendido na área de $1 \mathrm{~m}^{2}$ foi coletado com auxílio de espátula. As amostras foram homogeneizadas e secas a $60^{\circ} \mathrm{C}$ em estufa com circulação for çada de ar, moídas e a fração menor que 2,0 mm foi armazenada para análise posterior.

Nas amostras de solo, foram determinados os teores de carbono orgânico, por meio da oxi dação com dicromato de potássio em presença deácido sul fúrico e titulação do excesso de dicromato com sulfato ferroso amoniacal; N-total, pela digestão do sol o com ácido sulfúrico e água oxigenada seguida de destilação a vapor (Kjeldahl) com hidróxido de sódio e titulação do destilado coletado em indicador de ácido bórico com ácido sulfúrico diluído; $\mathrm{pH}$ em solução de $\mathrm{CaCl}_{2}$ 0,01 mol L-1 na relação 1:2,5 (sol o:sol ução); Ca, Mg e Al extraídos com solu ução de $\mathrm{KCl} 1$ mol L-1 na relação 1:10 (solo:sol ução), sendo Ca eMg determinados por espectrofotometria deabsorção atômica e Al por titulação com $\mathrm{NaOH} 0,015 \mathrm{~N}$, utilizando o indicador azul de bromotimol; acidez potencial $(\mathrm{H}+\mathrm{Al})$ pelo pH em SMP, a partir da curva de calibração do pH SMP versus H + Al para os solos do Paraná eK extraído com solução de Mehlich-1 $(\mathrm{HCl}$ 0,05 mol L-1 $+\mathrm{H}_{2} \mathrm{SO}_{4}$ 0,025 mol L-1) edeterminado por fotometria de chama (Pavan et al., 1992).

A avaliação dos efeitos dos sistemas de rotação de culturas sobreas características quími cas do solo foi realizada pela análise de variância, seguindo um delineamento de blocos casualizados, com quatro repetições, em cada profundidadedosol o. A diferença entre as médias de tratamentos foi avaliada pelo teste de Tukey a $5 \%$.

\section{RESULTADOS E DISCUSSÃO}

Após seteanos decultivo em diferentes sistemas de rotação e sucessão de culturas, algumas características quími cas dos sol os foram signi ficativamente alteradas (Figuras 1 e 2). Em geral, os sistemas TS (trigo-soja em semeadura direta) e TM (tremoçomilho-trigo-soja em semeadura di reta) apresentaram comportamentos extremos em relação aos sistemas TSA (trigo-soja-aveia-soja em semeadura direta, principalmente) e TMA (tremoço-milho-aveia-sojatrigo-soja em semeadura direta, principalmente).

A acidificação do solo, caracterizada pela diminuição do pH e do teor de Ca trocável eaumento da acidez trocável $(\mathrm{Al})$ e potencial $(\mathrm{H}+\mathrm{Al})$, e o aumento do teor de $\mathrm{N}$-total, no sistema TM, foram as principais alterações químicas observadas (Figuras 1 e 2 e Quadro 2). No caso do Ca, considerando que a diminuição de $1 \mathrm{cmol}_{\mathrm{C}} \mathrm{kg}^{-1} \mathrm{deCa}$ requer a aplicação de $1.000 \mathrm{~kg}$ ha-1 de $\mathrm{CaCO}_{3}$ para sua reposição, os resultados demonstraram que, em Londrina, a diminuição do teor de Ca nos sistemas com TSA, TMA e TM foi igual ou superior à do calcário aplicado em 1991 (2.000 kg ha-1). E m Campo Mourão, o teor de Ca foi equivalente ao do cal cário aplicado no mesmo período (1.700 kg ha-1) apenas no sistema TM.

\section{Quadro 2. Teores de Cálcio na camada de 0-20 cm e variação em relação ao valor inicial após sete anos sob diferentes sistemas de rotação de culturas em Londrina e Campo Mourão}

\begin{tabular}{|c|c|c|c|c|}
\hline \multirow{2}{*}{ Sistema } & \multicolumn{2}{|c|}{ Londrina } & \multicolumn{2}{|c|}{ Campo Mourão } \\
\hline & Cálcio & Diferença(2) & Cálcio & Diferença \\
\hline & & $\mathrm{cmol}_{\mathrm{c}}$ & $g^{-1}$ & - \\
\hline Inicial & 8,01 & & 3,48 & \\
\hline TS & $7,24(1)$ & $-0,77$ & 3,97 & $+0,49$ \\
\hline TSA & 5,78 & $-2,23$ & 3,75 & $+0,27$ \\
\hline TMA & 5,96 & $-2,05$ & 3,49 & 0,00 \\
\hline TM & 5,03 & $-2,98$ & 1,88 & $-1,60$ \\
\hline
\end{tabular}

\footnotetext{
(1) média do teores de Ca nas camadas de 0-10 e 10-20 cm (figuras 1 e 2). ${ }^{(2)}$ diferença entre teor de Ca no sistema após sete anos e o teor de $\mathrm{Ca}$ inicial.
} 
pH

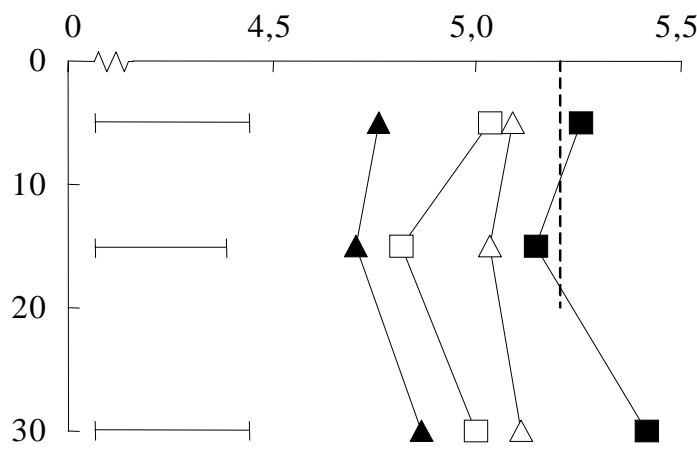

K, cmol $_{\mathbf{c}} \mathbf{k g}^{-1}$

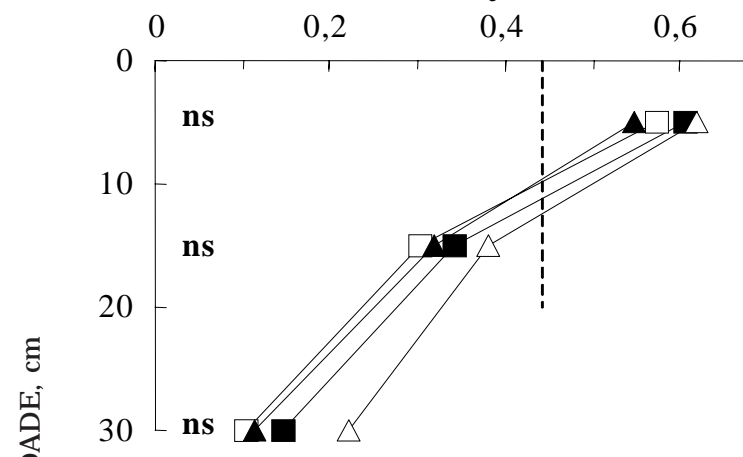

$\mathrm{H}+\mathrm{Al}, \mathrm{cmol}_{\mathrm{c}} \mathrm{kg}^{-1}$

3

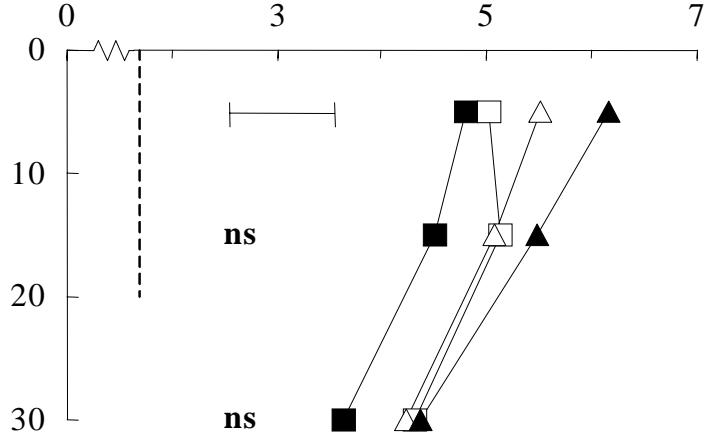

C orgânico, $\mathrm{g} \mathrm{kg}^{-1}$

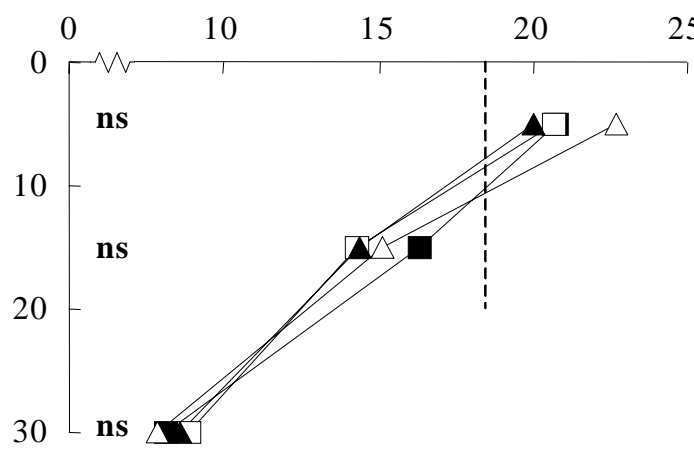

Al, $\mathrm{cmol}_{\mathrm{c}} \mathrm{kg}^{-1}$

0,1

0,2

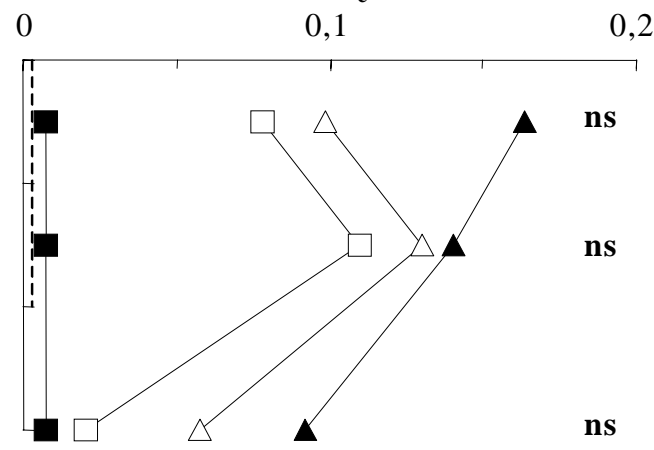

Ca, $\mathrm{cmol}_{\mathrm{c}} \mathrm{kg}^{-1}$
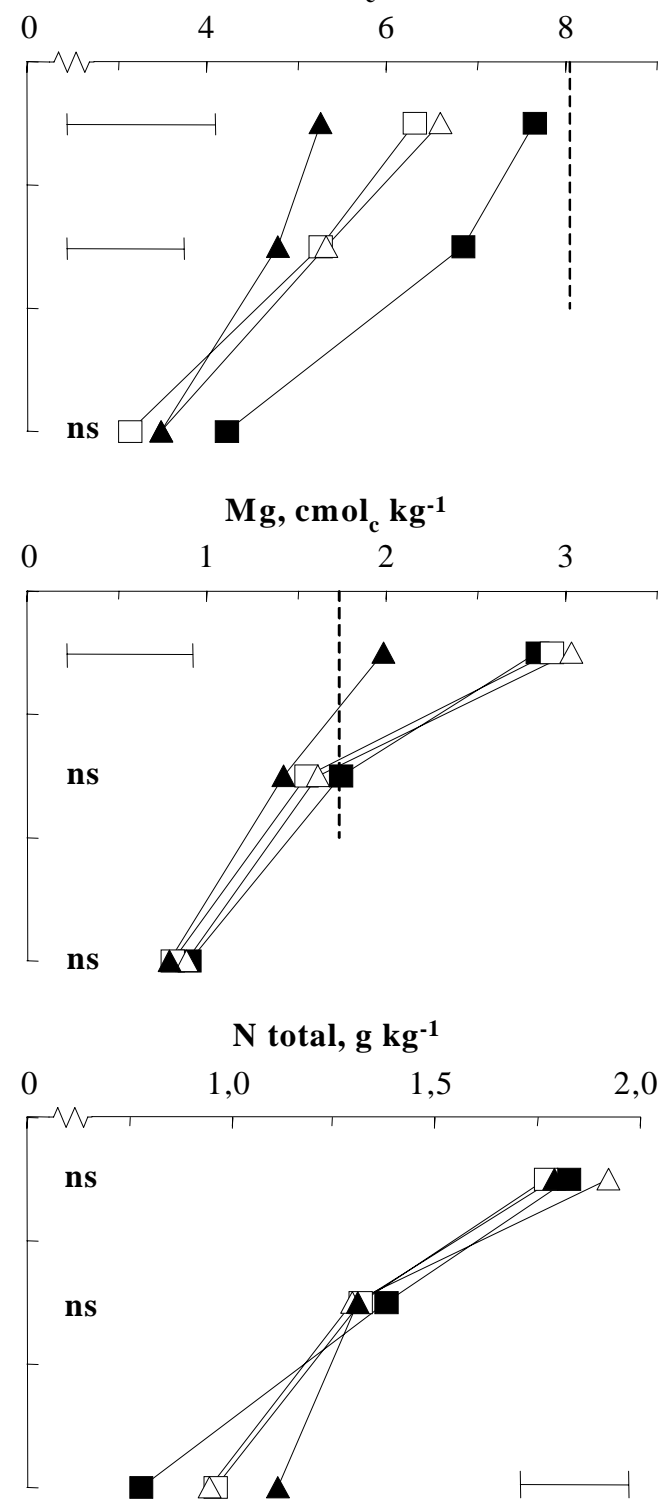

Figura 1. Características químicas do solo em Londrina. Tratamentos: TS ( $\square$ ); TSA( $\square)$; TMA( $\Delta)$; TM( $(\Delta)$; Valor Inicial (0-20 cm)(---). A seqüência de culturas utilizadas nos sistemas encontra-se no quadro 1. Os valores iniciais não foram considerados na análise estatística. Barras horizontais representam a diferença mínima significativa indicada pelo teste Tukey. 

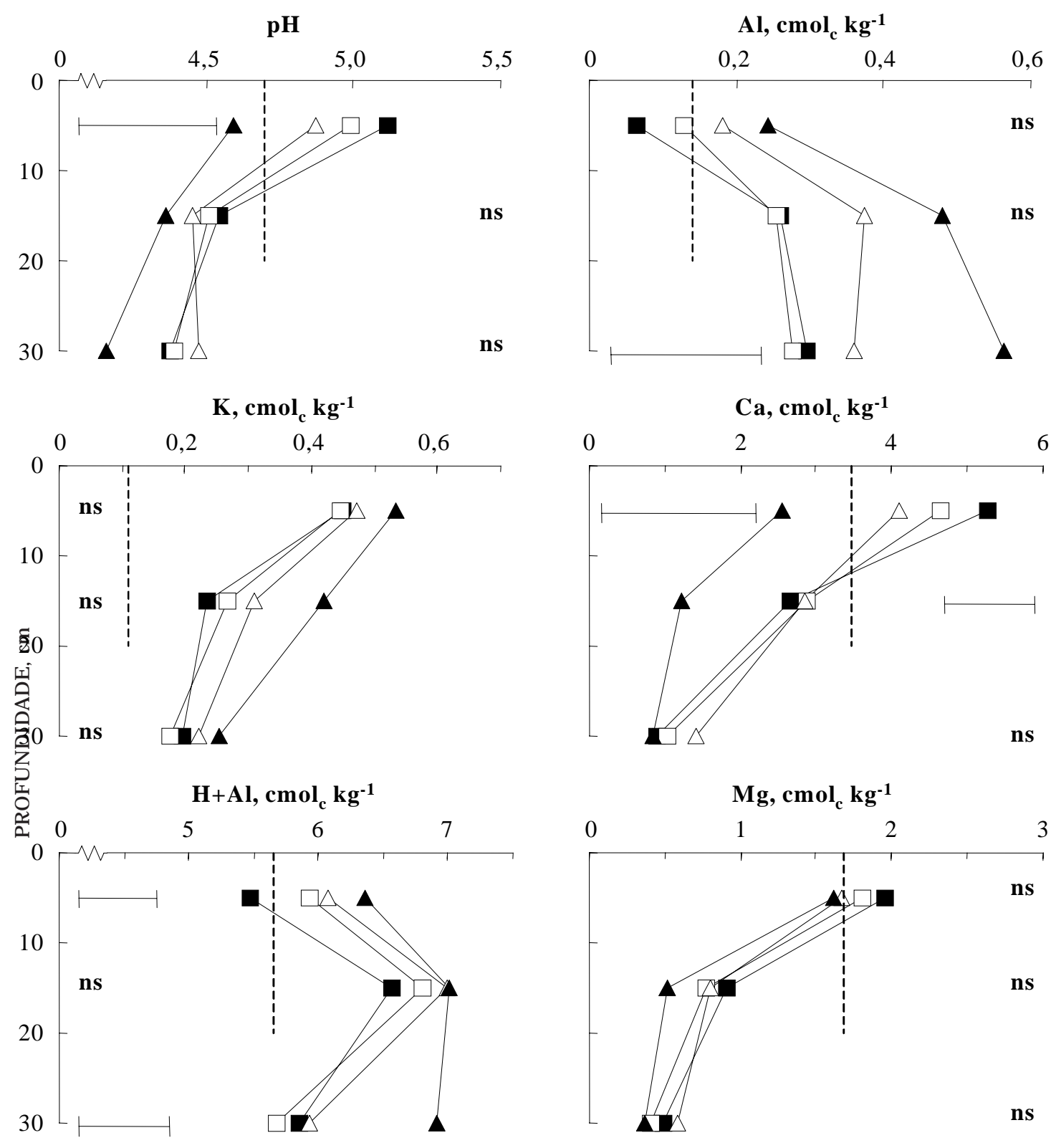

C orgânico, $\mathrm{g} \mathrm{kg}^{-1}$
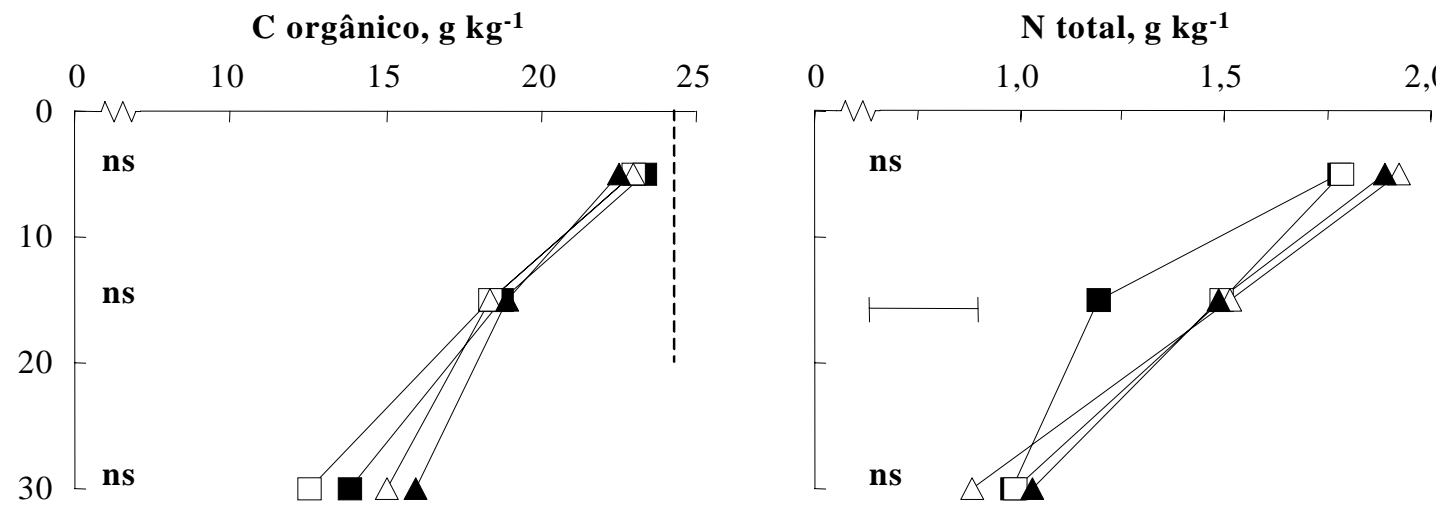

Figura 2. Características químicas do solo em Campo Mourão. Tratamentos: TS ( $\square$ ); TSA( $\square)$; $\operatorname{TMA}(\triangle)$; TM( $\Delta$ ); Valor Inicial $(0-20 \mathrm{~cm})(---)$. A seqüência de culturas utilizadas nos sistemas encontra-se no quadro 1. Os valores iniciais não foram considerados na análise estatística. Barras horizontais representam a diferença mínima significativa indicada pelo teste Tukey. 
A adubação nitrogenada com fontes amoniacais tem sido indicada como uma das principais causas da acidificação da superfície do solo no sistema de semeadura direta, graças à produção de prótons durantea nitrificação doamônio (Blevins et al., 1977; Helyar, 1991; Bolan et al., 1991). Neste contexto, Paiva et al. (1996) observaram quea aplicação anual de $90 \mathrm{~kg}$ ha-1 de $\mathrm{N}$ na forma de sulfato deamônio na sucessão milho/tremoço requereu $3.000 \mathrm{~kg} \mathrm{ha}^{-1}$ de cal cário para o restabelecimento do pH inicial do solo após sete anos sob o sistema. Raij et al. (1982) verificaram, em ensaio de calagem para o milho recebendo $100 \mathrm{~kg} \mathrm{ha}^{-1}$ de $\mathrm{N}$ como sulfato de amônio, que, após cinco anos, as perdas de Ca e M g chegaram a representar $1,22 \mathrm{cmol}_{\mathrm{C}} \mathrm{kg}^{-1} \mathrm{ano}^{-1}$.

No presenteestudo, ototal de $\mathrm{N}$ aplicadona forma desulfato deamônio, considerando o trigo e o milho, foi de 320, 160, 300 e $440 \mathrm{~kg}^{2} \mathrm{a}^{-1}$, para os sistemas TS, TSA, TMA eTM, respectivamente. Teoricamente, seriam necessários 1.500; 700; 1.400 e $2.100 \mathrm{~kg} \mathrm{ha}^{-1}$ de $\mathrm{CaCO}_{3}$, respectivamente, para neutralizar os prótons produzidos pela completa nitrificação do N aplicado. Neste caso, obteríamos a seguinte ordem de acidificação nos diferentes sistemas: TM >TS > TMA >TSA, diferenteda observada: TM $>$ TMA $\geq$ TSA $>$ TS. Deste modo, além do uso de sulfato de amônio, outra fonte de acidificação deve ser consi derada nos sistemas, principalmente naqueles envolvendo plantas de cobertura-adubação verde leguminosas.

No caso do tremoço, a redução do $\mathrm{pH}$ pode ser atribuída à liberação de protóns durante o processo de fixação biológica do $\mathrm{N}$ em virtude da dissociação de grupos carboxílicos de aminoácidos, para a manutenção da el etroneutralidade na célula (Marschner \& Hömheld, 1983; Bolan et al., 1991; Coventry \& Slattery, 1991). A quantidade de prótons liberada é equivalente ao excesso de absorção de cátions sobre ânions em leguminosas e varia entre 0,2 e 0,7 mol de $\mathrm{H}^{+}$por mol de $\mathrm{N}$ fixado (Nyatsanga \& Pierre, 1973; Bol an et al., 1991). Deveser ressaltado que, no sistema TM, o tremoço no inverno apenas compensaria a ausência da soja noverão, substituída pel o milho, o que teoricamente anularia este efeito, a menos que o potencial de acidificação da soja e o do tremoço sejam diferenciados. A classificação do tremoço no grupo de plantas com potencial médio de acidificação da rizosfera eda soja no grupo de plantas de baixo potencial ou indiferentes quanto à mesma característica, feita por Römheld (1986) confirma esta hipótese ejustifica a ocorrência de aci dificação dosol o em sistemas deculturas envol vendo o tremoço (Coventry \& Slattery, 1991; Paiva et al.,1996).

Foi observado um aumento do teor de $\mathrm{N}$ nas camadas de $10-20 \mathrm{~cm}$ e de $20-40 \mathrm{~cm}$ no sistema TM, em Campo Mourão e Londrina, respectivamente (Figuras 1 e 2), concordando com os aumentos de $\mathrm{N}$ nas camadas mais profundas do sol o observados por Reeves et al. (1984) eH einzmann (1985) em sistemas envolvendo o tremoço. Este aumento do teor de $\mathrm{N}$ em camadas subsuperficiais no sistema TM indica a lixiviação do N nitrificado, o que explicaria a acidificação da camada superficial do sol o. Para que o nitrato seja lixiviado, cátions básicos (K, Ca ou Mg) são requeridos como íons acompanhantes, enquanto os prótons produzi dos pela nitrificação do amônioe, ou, do N orgânico permanecem na camada superficial como acidez potencial (Helyar, 1991; Bolan et al., 1991). A lixiviação de Ca como acompanhante do nitrato responderia por parte da diminuição do teor deste el emento no solo, embora seria esperado que o $\mathrm{K}$ fosse o cátion preferentemente lixiviado, considerando a menor energia de ligação com o complexo de troca (Loyola \& Pavan, 1989).

Neste sentido, Liu \& Hue (1996) demonstraram que fulvatos de Ca derivados de resíduos vegetais, considerando a estabilidade do complexo orgânico, apresentam elevada mobilidade em solos ácidos, evidenciando o potencial para a melhoria das condições químicas do subsolo com a utilização de resíduos vegetais. Este mesmo comportamento foi observado em experimentos de campo em sistemas de semeadura direta onde o cal cário foi aplicado na superfície do solo (Sidiras \& Pavan, 1985; Oliveira \& Pavan, 1996; Caires et al., 1998; 1999).

A redução dos teores de Ca na camada arável do solo no sistema TM demonstra que, após sete anos sob esta rotação de culturas, a lixiviação excedeu o Ca aplicado. Neste caso, deve ser considerado que o tremoço em sistemas de rotação em SD apresenta grande potencial para a mobilização de $\mathrm{Ca}$ em solo ácido graças à formação de pares de íons ligantemetal ou de complexos solúveis ligante-metal entre Ca e compostos orgânicos de baixo peso molecular (RO- e RCOO-) (Harter \& Naidu, 1995; Aoyama, 1996a; 1996b) liberados durantea sua decomposição. Na forma orgânica, o Ca teria sua carga líquida alterada, reduzindo a energia de ligação com o complexo de troca, o que favorece sua lixiviação e a manutenção do K no solo. A ausência de diferença entre os teores de $\mathrm{K}$ no sol o nos diferentes sistemas confirma este comportamento.

A rotação de culturas em semeadura direta é um dos sistemas de produção mais indicados para o manejo de sol os ácidos. No entanto, deve-se chamar a atenção para o fato de o aumento das perdas de Ca em al guns sistemas requerer maior reposição do elemento pela aplicação de cálcario ou redução do interval o de tempo entre as aplicações de corretivo; caso contrário, culturas mais sensíveis à acidez poderão ter sua produtividade afetada.

\section{CONCLUSÕES}

1. As perdas de Ca trocável podem ser superiores às do Ca aplicado como cal cário, principal mente em sistemas onde as leguminosas são usadas como plantas de cobertura-adubação verde. 
2. O uso de maior quantidade de fonte de $\mathrm{N}$ na forma amoniacal e a presença do tremoço em sistemas de rotação de culturas provocam a aci dificação do solo, caso em que o interval o de tempo entre as aplicações de cal cário nestes sistemas deve ser reduzido, para recuperar os teores de Ca trocável e elevar o $\mathrm{pH}$.

\section{AGRADE CIMENTOS}

Aos funcionários do Laboratório de Solos da Embrapa Soja, Rinaldo B. Conceição, Fábio Ortiz, Valdemar de Oliveira Neto, Nelson Avila Simão, Moisés de Aquino e N estor Rioti Miura, pelo auxílio técnico nas análises de solo. Aos Técnicos Agrícolas, J osé Zucca Morais e Rubson N.R. Sibaldelli, pelo auxílio nos experimentos de campo.

\section{LITERATURA CITADA}

AOYAMA, M. Fractionation of water-soluble organic substances formed during plant residue decomposition and high performancesize exclusion chromatography of thefractions. Soil Sci. Plant Nutr., 42:21-30, 1996a.

AOYAMA, M. Use of high performance size exclusion chromatography to monitor the dynamics of water soluble organic substances during the decomposition of plant residues in soil. Soil Sci. Plant Nutr., 42:31-40, 1996b.

BAYER C. \& MIELNICZUK, J. Nitrogênio total de um solo submetido a diferentes métodos de preparo e sistemas de cultura. R. Bras. Ci. Solo, 21:235-239, 1997.

BLEVINS, R.L.; THOMAS, G.W. \& CORNELIUS, P.L. Influence of no-tillage and nitrogen fertilization on certain properties after 5 years of continuous corn. Agron. J ., 69:383-386, 1977.

BOLAN, N.S.; HEDLEY, M.J . \& WHITE, R.E. Processes of soil acidification during nitrogen cycling with emphasis on legume based pastures. In: WRIGHT, R.J .; BALIGAR, V.C. $\&$ MURRNAN, R.P., eds. Plant-soil interactions at low pH. Dordrecht, Kluwer Academic, 1991. p.169-179.

CAIRESE.F.; CHUEIRI, W.A.; MADRUGA, E.F.\& FIGUEIREDO, A. Alterações de características quími cas do sol o e resposta da soja ao calcário e gesso aplicados na superfície em sistema de cultivo sem preparo do solo. R. Bras. Ci. Solo, 22:27-34, 1998

CAIRESE.F.; FONSECA, A.F.; MENDES, J .; CHUEIRI, W.A.\& MADRUGA, E.F. Produção de milho, trigo e soja em função das alterações das características químicas do solo pela aplicação de cal cário e gesso na superfície, em sistema de plantio direto. R. Bras. Ci. Solo, 23:315-327, 1999.

COVENTRY, D.R. \& SLATTERY, N.J . Acidification of soil associated with lupines grown in crop rotation in Northeastern Victoria. Aust. J. Agric. Res., 42:391-397, 1991.

DERPSCH, R.; SIDIRAS, N. \& HEINZMANN, F. Manejo do solo com coberturas verdes de inverno. Pesq. Agropec. Bras., 20:761-773, 1985.
GONZALEZ-ERICO, E.; KAMPRATH, E.J .; NADERMAN, G.C. $\&$ SOARES, W.V. Effect of depth of lime incorporation on the growth of corn on an Oxisol of Central Brazil. Soil Sci. Soc. Am. J ., 45:1155-1158, 1979.

HARTER, R.D. \& NAIDU, R. Role of metal-organic complexation in metal sorption by soils. Adv. Agron., 55:219-263, 1995.

HEINZMAN N, F.X. Resíduos culturais de inverno e assimilação de nitrogênio por culturas de verão. Pesq. Agropec. Bras., 20: 1021-1030, 1985.

HELYAR, K.R. The management of acid soils. In: WRIGHT, R.J .; BALIGAR, V.C.; MURRNAN, R.P., eds. Plant-soil interactions at low pH. Dordrecht, Kluwer Academic Publishers, 1991. p.365-382.

KEMPER, B. \& DERPSCH, R. Results of studies made in 1978 and 1979 to control erosion by covers crops and no-till tillage techniques in Paraná, Brazil. Soil Till. Res., 1:253-267, 1981.

LIU, J . \& HUE, N.V. Ameliorating subsoil acidity by surface application of calcium fulvates derived from common organic materials. Biol. Fertil. Soils, 21:264-270, 1996.

LOYOLA J r, E. \& PAVAN, M.A. Seletividade de troca de cátions em solos ácidos. R. Bras. Ci. Solo, 13:131-138, 1989.

MARSCHNER, H. \& HÖMHELD, V. In vivo measurement of rootinduced $\mathrm{pH}$ changes at the soil-root interface: Effect of plant species and nitrogen source. Z. Pflanzenphysiol., 111:241251, 1983.

MUZILLI, O.; VIEIRA, M.J .; ALMEIDA, F.L.S.; NAZARENO, N.R.X.; CARVALHO, A.O.R.; LAURENTI, A.E. \& LLANILO, R.F. Comportamento e possibilidades da cultura do milho em plantio direto no estado do Paraná. Pesq. Agropec. Bras., 18:41-47, 1983.

NYATSANGA, T. \& PIERRE, W.H. Effect of nitrogen fixation by legumes on soil acidity. Agron. J ., 65:936-940, 1973.

OLIVEIRA, E.L. \& PAVAN, M.A. Control of soil acidity in notillage system for soybean production. Soil Till. Res., 38:4757, 1996.

PAIVA, P.J .R.; VALE, F.R.; FURTINI NETO, A.E. \& FAQUIN, V. Acidificação de um Latossolo Roxo do estado do Paraná sob diferentes sistemas de manejo. R. Bras. Ci. Solo, 20:7175, 1996.

PAVAN, M.A.; BINGHAM, F.T. \& PRATT, P.F. Chemical and mineralogical characteristics of selected acid soils of the State of Paraná, Brazil. Turrialba, 35:131-139, 1985.

PAVAN, M.A.; BINGHAM, F.T. \& PRATT, P.F. Redistribution of exchangeable calcium, magnesium, and aluminum following lime and gypsum applications to a Brazilian Oxisol. Soil Sci. Soc. Am. J ., 48:33-38, 1984.

PAVAN, M.A.; BLOCH, M.F .; ZEMPULSKI, H.D.; MIYAZAWA, M. \& ZOCOLER, D.C. Manual de análise química do solo e controle de qualidade. Londrina, Instituto Agronômico do Paraná, 1992. 40p. (IAPAR. Circular, 76)

PÖTTKER, D. \& BEN,J .R. Calagem para uma rotação deculturas nosistema plantio direto. R. Bras. Ci. Solo, 22:675-684, 1998.

RAIJ , B. van; CANTARELLA, H.; CAMARGO, A.P. \& SOARES, E. Perdas de cál cio e magnésio durantecinco anos em ensaio de calagem. R. Bras. Ci. Solo, 6:33-37, 1982. 
REEVES, T.G.; ELLINGTON, A. \& BROOKE, H.D. Effects of lupin-wheat rotations on soil fertility, crop disease and crop yields. Aust. J . Exp. Agric. Anim. Husb., 24:595-600, 1984.

RHEINHEIMER, D. S.; KAMINSKI, J .; LUPATINI, G. C. \& SANTOS, E.J.S. Modificações em atributos quími cos de solo arenoso sob sistema plantio direto. R. Bras. Ci. Solo, 22:713721, 1998.

RITCHEY, K.D.; SILVA, J .E. \& COSTA, U.F. Calcium deficiency in clayey B horizons of Savannah Oxisols. Soil Sci., 133:378382, 1982.
RÖMHELD, V. pH changes in the rhizosphere of various crop plants, in relation to the supply of plant nutrients. PotashReview, Subject 6, 55th Suite, 12:1-12, 1986.

SIDIRAS, N. \& PAVAN, M .A. I nfluência do sistema de manejo do solo no seu nível defertilidade. R. Bras. Ci. Solo, 9:249-254, 1985.

SIDIRAS, N.; HENKLAIN, J .C. \& DERPSCH, R. Comparison of three different tillage system with respect to aggregate stability, the soil and water conservation and the yields of soybean and wheat on an oxisol. J . Agron. Crop Sci., 151:137148, 1982. 
J. C. FRANCHINI et al.

R. Bras. Ci. Solo, 24:459-467, 2000 